TRABAJOS DE PREHISTORIA

53, n. ${ }^{\circ} 1,1996$, pp. 5-13

\section{EL ESTUDIO DE LA PREHISTORIA DEL VIEJO MUNDO EN LOS ESTADOS UNIDOS DE AMÉRICA}

\author{
THE STUDY OF OLD WORLD \\ PREHISTORY IN THE UNITED STATES \\ OF AMERICA
}

\section{LAWRENCE G. STRAUS (*)}

\section{RESUMEN}

La enseñanza y el estudio de la Prehistoria del Viejo Mundo están difundidos en los Estados Unidos. Sin embargo, la verdadera investigación (especialmente la excavación) está mucho más restringida en las numerosas instituciones que tienen supuestos especialistas en algunos aspectos de este campo académico. Hay bastante interés principalmente por el Paleolítico/Mesolítico de Europa y de África, generalmente asociado con una perspectiva paleoantropológica. Hay también numerosos investigadores norteamericanos ocupados en el estudio de la Prehistoria final/Protohistoria del Oriente Medio y Europa, con menos especialistas en el Neolítico per se. Este artículo expone muy brevemente la formación, financiación, organización, publicación y enfoques teóricos de la arqueología prehistórica en los EE. UU. Subraya las razones por las cuales los investigadores norteamericanos se acercan al estudio del registro del Viejo Mundo desde unos puntos de vista diferentes pero sin embargo complementarios a los de sus colegas europeos, africanos y asiáticos. Espera que exista todavía un lugar para la investigación cooperativa que incluya a los norteamericanos en asociación plena con sus pares del Viejo Mundo en este mundo post-colonial y de la post-Guerra Fría.

(*) Departamento de Antropología. Universidad de Nuevo México. Albuquerque, NM 87131 USA.

El artículo fue remitido en su versión final el 20-II-96.

\begin{abstract}
The teaching and study of Old World Prehistory are widespread and growing in the United States. However, actual research (especially excavation) is far more restricted among the many colleges, universities and museums that have specialists in particular aspects of this broad field. There is considerable interest notably in the Paleolithic/Mesolithic of Europe and Afri$c a$, generally associated with a paleoanthropological perspective. American researchers in anthropology departments are also heavily engaged in studies of terminal prehistory/protohistory in the Near East and Europe, with fewer specialists in the Neolithic per se. This article discusses the training, financing, organization, publication and theoretical foci for Old World prehistoric archeology in the U.S. It highlights the reasons for which American researchers usually approach the study of the Old World record from points of view that are different from and yet complementary to those of their European, African and Asian colleagues, who, unlike the Americans, feel they are directly studying their own national past. There is hopefully still a place for cooperative research involving Americans in full partnership with their Old World peers in the post-colonialist, post-Cold War world.
\end{abstract}

Palabras clave: Prehistoria. Estados Unidos de America. Viejo Mundo. Investigación. Enseñanza. Financiación. Publicación. Arqueología. Paleoantropología. Paradigmas. 
Key words: Prehistory. United States of America. Old World. Research. Training. Financing. Publication. Archeology. Paleoanthropology. Paradigms.

\section{INTRODUCCIÓN}

Lo que sigue es una nota breve e informal sobre el estado de la investigación y de la enseñanza de la Prehistoria del Viejo Mundo en los principales centros académicos y museos de los Estados Unidos de América, con un énfasis especial en lo relativo al Paleolítico de Europa. No tiene la pretensión de ser ni completa ni detallada, sino de dar una visión rápida de la situación actual desde el punto de vista de alguien que ha participado activamente en este mundo norteamericano del estudio de la Prehistoria del Viejo Mundo desde hace un cuarto de siglo. Se trata de pasar revista a la importancia relativa de los investigadores norteamericanos que trabajan en Europa, África, Asia occidental y oriental, a los períodos de su interés, a las instituciones y fundaciones que apoyan y financian sus trabajos, a la naturaleza de la enseñanza de la Prehistoria en los EE.UU., etc. Una revisión de este tipo no puede tener completamente en cuenta la gran variabilidad que existe en varios de los campos descritos, pero intentaré presentar al menos un esquema actualizado del panorama nacional tal como lo veo yo en 1996.

\section{"LA PLANTILLA"}

La guía anual de la Sociedad para la Arqueología Americana (SAA) del año académico 1995-96 indica que un $15 \%$ de los 1.293 miembros de la Sociedad que respondieron a un cuestionario, expresaron "interés" por la arqueología del viejo mundo. La SAA tiene un total de $>5.600$ miembros y es la principal asociación arqueológica en los EE.UU. ¿Pero, qué quiere decir "interés"? Hay, evidentemente, una gran diferencia entre la enseñanza y la investigación en cuanto a la noción de "interés". Y dentro de la categoría de "investigación" hay una diferencia significativa entre los que realmente dirigen excavaciones en el Viejo Mundo y los que simplemente estudian las colecciones hechas por prehistoriadores del Viejo Mundo.

Para profundizar un poco más, hemos seleccionado una muestra de los 226 departamentos universitarios de Antropología (o de "Sociología y Antropología") más significativos en cuanto a su tamaño, fama de la universidad, etc. (hay un total de 417 departamentos universitarios de enseñanza antropológica en los EE. UU.) y de los 24 departamentos de investigación antropológica en museos (hay un total de 68 en el país). Esta muestra proviene de la guía anual de la Asociación Americana de Antropología (AAA) del año 1995-96, que da unas listas completas del personal docente e investigador de todas las instituciones antropológicas del país. No he incluido en mi muestra los departamentos canadienses (relativamente pocos, aunque muchos de ellos muy buenos) que están incluidos en la guía. Por supuesto, tampoco he incluido en este estudio "la otra arqueología": la clásica, dedicada a las civilizaciones de Roma, Grecia, Egipto y Mesopotamia, y que tiene sus departamentos propios (normalmente, en asociación con las lenguas clásicas), con revistas y fuentes de financiación propias, etc. Hay algunos individuos que estudian las civilizaciones clásicas desde la perspectiva antropológica y que trabajan en departamentos de Antropología, pero no están sistemáticamente incluidos aquí. Los departamentos de Antropología que no hemos seleccionado pertenecen a instituciones realmente de carácter local y de muy pequeña escala (muchas veces son solamente secciones de Antropología dentro de departamentos de ciencias sociales). Esta guía de la AAA provee información sobre las áreas de competencia y de interés de los profesores o investigadores de cada departamento.

Hemos dividido los 170 arqueólogos norteamericanos que se autodefinen como especialistas en el Viejo Mundo según cuatro categorías geográficas (África, Europa, Oriente Medio y Asia oriental) y según cuatro grandes períodos prehistóricos (Paleolítico-Mesolítico, Neolítico, Calcolítico-Bronce-Hierro, y "sin especificar"[y desconocido por mí]). Para el Paleo-Mesolítico hemos contabilizado 23 estudiosos que se declaran especialistas en África, 9 en Oriente Medio, 5 en Asia oriental y 33 en

T. P., 53, n. ${ }^{\circ} 1,1996$ 
Europa. Hay, además, 6 individuos que trabajan en 2 ó 3 de estos continentes o grandes regiones. Para el Neolítico hay unos 6 que trabajan en Oriente Medio y otros 6 en Europa (algunos de los neolitistas en ambas regiones también trabajan en Paleolítico o Epipaleolítico). Para el final de la Prehistoria hay unos 6 para África, 17 para Oriente Medio, 3 para Asia oriental y 10 para Europa. En varios casos, los que trabajan en Paleo-Mesolítico también trabajan en el Neolítico, pero rara vez se solapan las Edades de Piedra con las de los Metales. En algunos casos, los individuos son especialistas (notablemente en la arqueozoología) en varias épocas y regiones. Hay varios individuos que indican su interés geográfico, pero sin especificar época (y su posible especialidad es desconocida por mí): 7 para África, 26 para Oriente Medio (la gran mayoría seguramente interesada en las épocas más recientes), 9 para Asia oriental y 10 para Europa (que, desde luego, no son paleolitistas). Finalmente, hay 6 que no especifican ni región del Viejo Mundo ni época. Probablemente, muchos de estos dos últimos grupos (y seguramente algunos de los otros) se interesan por y enseñan algo sobre la Prehistoria del Viejo Mundo, pero no hacen investigación propia -especialmente trabajos de campo-fuera de los EE.UU. Sin embargo, como pueden ver, son números relativamente importantes, especialmente para el Paleo-Mesolítico de Europa y Africa y (aunque bastante menos) también para las Edades de los Metales en Oriente Medio y Europa.

\section{LA FORMACIÓN ACADÉMICA}

Es importante darse cuenta que (a diferencia de un país como España, que todavía tiene un sistema de educación superior bastante centralizado) Estados Unidos tiene un sistema totalmente autónomo; cada Universidad (o estado) establece sus propias normas, curricula, y reglamentos. Lo que sigue intenta describir el tipo "promedio" de formación que puede recibir un alumno de Prehistoria en un buen centro. Mi propia experiencia ha sido algo anormal para un norteamericano: he hecho todos mis estudios universitarios en una Universidad privada (la de Chicago - pero con un semestre como "investigador visitante" en una universidad estatal, la de Michigan-) y he sido profesor solamente en una Universidad estatal, la de Nuevo México. La mayoría de los alumnos y de los profesores norteamericanos se mueve bastante entre Universidades, e incluso los alumnos cambian de carrera, especialmente entre "college" y "graduate school" (es decir, entre sus primeros cuatro años de Universidad ["Bachelor"] y sus años de estudios superiores ["Master" y" Doctor"]).

La carrera universitaria norteamericana completa de Prehistoria incluye tres diplomas: "Bachelor", "Master" y "Doctor" — normalmente en "artes" o "filosofía, pero a veces en "ciencias", según el programa específico, y casi siempre se hace en un departamento de Antropología (y no en Historia como en España). Recientemente, algunos programas de Arqueología se han independizado completamente para crear departamentos propios, como en el caso de la Universidad de Boston (y como es generalmente el caso en Canadá), pero más "normal" es la existencia formalizada de una sección de Arqueología con más o menos autonomía dentro de un departamento de Antropologíacomo es el caso en las Universidades de New Mexico, Arizona, Chicago, etc.- En algunos casos, como Harvard, Michigan, Pennsylvania, Kansas, etc., los profesores y alumnos de Prehistoria están ligados administrativamente a departamentos de Antropología, pero se hallan separadamente en museos de Arqueología. Estas secciones de Arqueología determinan sus propios curricula y requisitos dentro de las normas de cada departamento y Universidad.

El primer título universitario (cuatro años, en principio), el de "Bachelor", puede incluir una especialidad en Antropología con una sub especialización en Arqueología. Normalmente, depués de 2-3 años de cursos de formación general post secundaria (letras, ciencias sociales, físicas y biológicas), el alumno interesado en la Antropología o, más específicamente en la Arqueología, recibe clases en las varias ramas de la Antropología (socio-cultural, bio-cultural, físico-biológica, lingüística y arqueológica). Puede también empezar la acumulación de ex- 
periencia práctica en escuelas de campo veraniegas o incluso, después de cierto tiempo, en la Arqueología contractual. Terminado el diploma de "Bachelor", el alumno puede empezar su formación profesional. De ordinario, solicita la admisión en otra Universidad para cambiar de ambiente, para oír otros puntos de vista, para obtener instrucción distinta o más especializada, etc. A la vez, busca una beca a nivel nacional (muy escasas) o a nivel de la Universidad donde sea candidato (las becas son muy variables entre las Universidades ricas y pobres). A falta de beca, muchos alumnos reciben préstamos del gobierno y consiguen trabajos dentro de la Universidad que están subvencionados por el gobierno.

Normalmente el programa de "Master" en Arqueología (que es bastante variable en contenido y en requerimientos de un centro a otro centro) cuesta un mínimo de dos años, con una mezcla de clases obligatorias y optativas en diferentes aspectos de la Arqueología (y también Antropología cultural y biológica, estadística, geología, etc.). Habitualmente el curso termina con un examen y/o una tesina. Si el alumno decide quedarse en el mismo centro para hacer un doctorado, a veces el examen de "Master" sirve también como prueba para la entrada a la candidatura al doctorado.

Ahora, el alumno escoge un comité doctoral (normalmente, un director de tesis y otros dos profesores) y prepara la especialidad y el enfoque de su tesis, con clases y cursos de lectura individualizados. En algunos departamentos hay un examen sobre los temas (teóricos, cronológicos, geográficos, etc.) relacionados con la tesis que propone el alumno. Este examen (y el anterior) puede ser escrito, oral o una combinación de las dos formas. Luego, el alumno tiene que escribir una propuesta de tesis que debe ser aceptada por su comité, por la facultad de Arqueología o, a veces, por la facultad entera de Antropología. A la vez, el alumno solicita subvenciones ante fundaciones gubernamentales y/o privadas para hacer la investigación de la tesis. La tesis es juzgada y aprobada por este comité (a veces con la adición de un lector de fuera del departamento o incluso de fuera de la Universidad en cuestión).
Por supuesto, todo esto es bastante más dificultoso para los alumnos norteamericanos que trabajan en el extranjero (especialmente en el Viejo Mundo) que para los que hacen sus tesis dentro de los EE.UU. y que tienen el acceso más fácil a las oportunidades de hacer prospecciones o excavaciones, de estudiar colecciones o de obtener becas o empleos arqueológicos a veces relacionados con el tema de sus tesis. Siendo grandes los obstáculos para los alumnos norteamericanos que quieren hacerse prehistoriadores del Viejo Mundo, son relativamente pocos los que terminan su doctorado en este campo. La gran mayoría de éstos está formada en unos pocos centros donde suele haber al menos un (o algunos) profesor(es) activo(s) en la investigación en el extranjero. El alumno doctoral norteamericano suele trabajar en las excavaciones de su "maestro" (o de los amigos de éste) y se beneficia de las relaciones que su profesor pueda tener con colegas en el Viejo Mundo para obtener acceso a colecciones para hacer su investigación doctoral.

Una vez terminada la redacción de la tesis (y el proceso total después de obtener el "Master" puede llevar de tres a diez años o más, según la rapidez del alumno y especialmente la financiación con la que cuenta ya que muchos tienen que trabajar para vivir por lo que emplean más tiempo en los análisis y en la redacción) el recién doctorado se mete en el mercado de trabajo. Éste es bastante limitado para un especialista en la Prehistoria del Viejo Mundo, pero como resultado del hecino de que las plazas están más o menos ocupadas en las Universidades y museos que tradicionalmente han tenido especialistas en el Viejo Mundo, con el tiempo, estos especialistas se extienden a centros más pequeños o sin tradición a través del país. Sin embargo, es todavía el caso que la gran mayoría de los doctorados en la Paleoantropología o Prehistoria del Viejo Mundo es ofertada por unas pocas Universidades, notablemente Arizona, Arizona State, Berkeley, Boston, Chicago, Harvard, Michigan, New Mexico, New York, Southern Methodist (Dallas), Yale, etc. En los últimos años se han creado excelentes nuevos centros para la enseñanza de la Prehistoria paleolítica de África en las Universidades de Indiana y Rutgers (New Jersey),

T. P., 53, n. ${ }^{\circ} 1,1996$ 
para la de Europa en la Universidad de Pensylvania y para la del Viejo Mundo en general en la Universidad de Illinois. Y, desde luego, hay otros programas excelentes (pero más pequeños), con investigación activa en varias partes del Viejo Mundo, en varias otras Universidades del país, especialmente cuando se incluyen los programas de Paleontología humana (que no constan en los sumarios citados arriba). Pero la producción anual de doctorados en Prehistoria del Viejo Mundo es todavía limitada: 13 en un total de 400 doctorados en Antropología en el año académico 1993-94 (cosa afortunada, dada la escasez de puestos de trabajo hoy en día).

\section{LA FINANCIACIÓN}

Desde luego, es bastante más caro hacer investigación prehistórica en el Viejo Mundo desde una base en EE.UU. que desde el mismo país donde se encuentran los yacimientos. Además de los billetes de avión, puede haber más gastos para la vivienda y para el transporte del investigador y de sus alumnos en el extranjero. Es un tipo de investigación costoso en relación con la arqueología nacional.

Las investigaciones prehistóricas norteamericanas en el extranjero están subvencionadas por varias fuentes de financiación. Dos son del gobierno nacional y sus becas (las más importantes económicamente) son altamente competitivas: National Science Foundation y National Endowment for the Humanities. La NSF subvenciona generalmente los proyectos de carácter más bien "científico" y se concentra especialmente en las épocas más antiguas (orígenes del hombre, Paleolítico, Mesolítico, Neolítico). La NEH, por el contrario, subvenciona una Arqueología de carácter más bien humanístico, lo que quiere decir en la práctica las investigaciones de épocas más recientes (Edades de los Metales y altas civilizaciones). A veces, estas becas son para más de un año (hasta tres) y pueden cubrir los gastos no solamente de las excavaciones o prospecciones (y de los análisis arqueológicos), sino también de muchos estudios complementarios. Son difíciles de conseguir. Ambas fundaciones federales (y especial- mente la segunda) corren el peligro de sufrir importantes recortes de presupuesto (o incluso su eliminación) en el momento en que escribo, a raí de los cambios políticos en Washington.

También existen algunas fundaciones o sociedades privadas que subvencionan (entre otras cosas) la investigación prehistórica en el Viejo Mundo: entre ellas las más importantes son la National Geographic Society, la WennerGren Foundation for Anthropological Research, la L.S.B. Leakey Foundation y la American Philosophical Society. También existen organizaciones como "Earth Watch" que proveen voluntarios (que suelen ser adultos que pagan su viaje, su estancia y una contribución para el funcionamiento del proyecto) para las excavaciones.

Las solicitudes para estas subvenciones privadas son documentos menos largos que las solicitudes para las becas del gobierno, pero todas exigen un planteamiento bien desarrollado apoyado en un contenido teórico y empírico sólido. En todo caso serán evaluados primero por varios lectores anónimos especializados (o menos especializados, pero siempre son arqueólogos en el caso de una solicitud de contenido arqueológico) y después por el comité de la fundación (o de la sección arqueológica de la fundación). Hay un juicio científico y otro económico según las posibilidades financieras de cada fundación y la cantidad de solicitudes anuales. Si ambos juicios son favorables, la suma (total o parcial) del presupuesto es pagada directamente a la institución del investigador, que a su vez entrega el dinero (o paga los gastos) al investigador.

Las becas federales incluyen, como suplemento estatutario, una subvención indirecta a las instituciones que puede variar (por contrato institucional y según sus costes de operación) de un adicional $25 \%$ a un $75 \%$ de la propia suma de la beca. Así el gobierno federal provee fondos para mantener la infraestructura de las Universidades, que de otra manera no dependen de Washington (ya que son estatales, privadas o religiosas-y siempre autónomas).

Finalmente, y con grandes diferencias entre Universidades ricas y pobres, existen fondos propios de investigación que, al menos, sirven para hacer excavaciones o estudios preliminares o para cubrir huecos en las becas de fuera 
cuando éstas han sufrido recortes presupuestarios. Hay becas a nivel nacional para visitas (largas y cortas) de académicos norteamericanos a centros extrajeros y vice versa (Fulbright Foundation), para visitas e investigaciones en colaboración con estudiosos de países (ex-) comunistas (International Research and Exchange Board), y para la participación de norteamericanos en reuniones o congresos en el extranjero (American Council of Learned Societies, Social Science Research Council, etc.). Algunos prehistoriadores han creado "institutos" o fundaciones de investigación propias con el apoyo de mecenas particulares.

\section{LOS ENFOQUES TEÓRICOS DE LOS PREHISTORIADORES AMERICANOS Y EL CONCEPTO DE "PROYECTO DE INVESTIGACIÓN”}

Existe una diferencia de perspectiva fundamental, a mi juicio, entre los prehistoriadores que trabajan "en casa" y los que vienen "de fuera" para investigar el pasado de una región, país o continente que no es el suyo. Por definición, los norteamericanos que hacen investigación prehistórica en el Viejo Mundo están estudiando cosas muy remotas a su propia existencia en todos los sentidos. De hecho, la Arqueología de América desde sus comienzos en el siglo XVIII (con los trabajos personales de Thomas Jefferson, por ejemplo) siempre ha sido el estudio de "otros", de aborígenes sin relación genética, histórica o cultural alguna con los estudiosos. Cuando los norteamericanos "vuelven" al Viejo Mundo para hacer investigación arqueológica, tampoco les quedan normalmente vínculos significativos con el pasado objeto de su estudio. Así, ellos no consideran que estén reconstruyendo su historia -ni siquiera su historia ficticia-. El norteamericano que trabaja en una región del Viejo Mundo no tiene una implicación sentimental, patriótica o política con el objeto de su estudio; los fines de su investigación pueden ser simplemente "científicos" (y, tal vez, "fríos") en comparación con los fines (explícitos o implícitos) de un prehistoriador "nativo". (Personalmente, soy un caso algo ambiguo, ya que por un lado mi madre es francesa y mi abuelo y bisabuelo eran prehistoriadores en el Suroeste de Francia, y por otro lado mi esposa es montañesa, aunque vivo y he sido formado principalmente en los EE.UU.).

No hay que olvidar que la arqueología prehistórica norteamericana forma parte de las ciencias sociales; en Europa forma parte de las humanidades. Es una distinción importante que trae consecuencias paradigmáticas.

Veo muy normal que un español trabajando en España, un israelí en Israel, un chino en la China, o un keniata en Kenia considere y plantee la Prehistoria de su país de manera distinta (y probablemente de modo más "histórico") que un norteamericano (o cualquier otro extranjero sin raíces en el país) que podría tener la perspectiva más "objetiva" de las ciencias sociales. En realidad, salvo en algunos casos contados - como el de los vascos (y a veces incluso discutibles) - los vínculos entre un prehistoriador nativo moderno y los seres prehistóricos de "su" país o región podrían ser casi o completamente tan remotos como los vínculos entre un prehistoriador norteamericano y estos últimos, pero este hecho no cambia la realidad de una diferencia de perspectiva que no se puede dejar escapar: la histórica frente a la sociológica. Esto no quiere decir en absoluto que los trabajos de unos o de otros sean por sistema más rigurosos o mejores; simplemente, tienen otra perspectiva distinta que tiene consecuencias prácticas en la manera de enfocar la investigación y (¡muy importante!) de buscar subvenciones. En Europa, me parece que está generalmente aceptado que es interesante y digna de financiación (si es que hay fondos y el prehistoriador que propone hacer un trabajo tiene la formación y el curriculum vitae adecuados - iy las relaciones sociopolíticas apropiadas!) cualquier investigación seria que pueda aportar datos a la comprensión de la ocupación humana de un territorio determinado y del desarrollo de la cultura a través de los siglos y milenios. En EE.UU., en cambio, no es evidente por sí mismo que haya un interés implícito en la excavación de un yacimiento, en la construcción o refinamiento de una secuencia tecnocronológica, en un reconocimiento territorial, etc. - especialmente fuera del país-. Toda propuesta de subvención (incluso las "pequeñas") tiene que suponer una contribución signi-

T. P., 53, n. ${ }^{\circ} 1,1996$ 
ficativa a la resolución de un problema general (evolucionista, ecológico, etc.). La problemática es esencial y las ideas tienen que ser geniales; pero los hechos son a veces secundarios. Una fundación norteamericana no quiere dar dinero para contribuir a la historia de larga duración (longue durée) de una región del Viejo Mundo porque sí. Esta postura (lógica en cierto sentido) hace que los prehistoriadores norteamericanos tengan que prometer en sus solicitudes y en sus publicaciones (que cuentan mucho a la hora de decidir quién va a recibir subvenciones) muchos más resultados de los que pueden obtener normalmente y de manera realista con cualquier proyecto de investigación, especialmente con uno a corto plazo (que suele ser el máximo que uno puede esperar, dada la situación financiera actual para la Arqueología). Hay un círculo vicioso. Un problema serio es la dificultad de llevar a cabo proyectos a muy largo plazo; en esto los "nativos" tienen una ventaja obvia. Por un lado, las fundaciones norteamericanas quieren resolver grandes problemas teóricos de la Prehistoria, pero por otro lado no dan dinero a largo plazo. Así, los prehistoriadores norteamericanos que trabajan en el extranjero tienen que hacer campañas de investigación relativamente largas e intensivas. Pero, no obstante, muchas veces solamente pueden conseguir muestras limitadas dentro de yacimientos o dentro de regiones. Desgraciadamente, no pueden permitirse el "lujo" de pasarse la vida conociendo muy a fondo el registro de una región.

Sin embargo, ya que el registro prehistórico es finito (y en peligro de desaparición), no está mal que un prehistoriador tenga que justificar de manera muy seria y algo general las razones por las cuales quiere, con la excavación, destrozar una parte de este registro (una parte de la herencia universal del pasado humano) para aumentar la suma de nuestros conocimientos generales sobre la evolución humana. ¡Pero siempre sin llegar a los extremos ridículos de tener que decir, por ejemplo, que cualquier sondeo o reconocimiento va a resolver los problemas de la Prehistoria del mundo!

El sistema norteamericano suele exigir la creación de proyectos de investigación con un comienzo y un fin. Y los plazos suelen ser muy cortos (un año o al máximo dos o tres). Esto quiere decir que el prehistoriador norteamericano tiene que tener resultados inmediatemente y tiene que publicarlos (por muy parciales y preliminares que sean) rápidamente para poder buscar más subvenciones. Vivimos de subvención en subvención; casi no hay instituciones como el CNRS en Francia donde uno puede ser investigador a tiempo completo y con fondos para la investigación. Casi todos vivimos de la enseñanza, lo cual significa también que los períodos para hacer investigación son muy limitados (es decir, la "vacación" de verano, y tal vez un año o medio año sabático cada siete años). Existen solamente algunos museos muy importantes (notablemente el Smithsonian) que tienen prehistoriadores cuyo trabajo es hacer investigación más o menos a tiempo completo y que tienen presupuestos para financiar una parte importante de esta investigación. Los museos universitarios - a veces grandes - son docentes y no suelen tener personal investigador a tiempo completo, sino conservadores y profesores. Casi no hay "institutos" como en el sistema arqueológico alemán, por ejemplo. Hay, evidentemente, consecuencias de este sistema que pueden ser malas. Pero, en el fondo, es bueno obligar a la publicación, la justificación y la divulgación por medio de la enseñanza de lo que uno hace en la investigación destructiva que es la excavación arqueológica.

\section{LA PUBLICACIÓN Y LA DIVULGACIÓN}

Es bastante difícil publicar monografías sobre excavaciones del Viejo Mundo en EE.UU.; no hay un gran mercado para ellas. Hay mayor interés en obras de teoría y de síntesis, actas de simposios, etc. Las series o editoriales principales que publican actualmente obras de Prehistoria del Viejo Mundo en el país incluyen Plenum Publishing, varias editoriales universitarias (por ejemplo, Chicago, Indiana, Harvard, California, Princeton, Arizona, Arizona State, New Mexico, Columbia) y del Smithsonian, algunas series particulares nuevas (International Monographs in Prehistory, Monographs in World Archaeology) y una serie oficial de la AAA (Archeological Papers of the American Anthropological Association). 
En general, las revistas arqueológicas prefieren publicar artículos generales con más énfasis en la teoría y en las síntesis; no suelen publicar descripciones simplemente empíricas. Tal vez, las excepciones son Journal of Field Archaeology y Journal of Archaeological Science (esta última es una revista anglo-americana). Las principales revistas norteamericanas de Antropología y de Arqueología publican artículos sobre la Prehistoria del Viejo Mundo con cierta regularidad: American Antiquity, American Anthropologist, Journal of Anthropological Research, Geoarchaeology, Quaternary Research, Journal of Anthropological Archaeology y especialmente Current Anthropology.

La Prehistoria del Viejo Mundo tiene cierta importancia en las reuniones anuales de la SAA, con simposios temáticos (escogidos entre propuestas detalladas hechas unos ocho meses antes de cada reunión del mes de abril) y sesiones generales (donde se agrupan comunicaciones individuales que habían sido escogidas aunque no hubieran sido invitadas dentro del marco de un simposio). De los 106 simposios y sesiones en la reunión de 1994 (jen Disneylandia!), hubo 11 (10\%) que trataron de varios aspectos, temas o regiones de la Prehistoria del Viejo Mundo, por ejemplo. De los cerca de 400 simposios y sesiones de la reunión anual de la AAA de 1995, solamente 4 trataron parcial o totalmente temas de la Prehistoria del Viejo Mundo. Hay también comunicaciones relevantes (de paleontología humana) en las reuniones anuales de la American Association of Physical Anthropologists (AAPA). Pero, hace cinco años se ha fundado la Paleoanthropology Society, un grupo relativamente informal y pequeño que agrupa a los paleoantropólogos y prehistoriadores paleolitistas de los EE.UU. No tiene revista, pero tiene una reunión anual, alternando entre las reuniones de la SAA y de la AAPA. Finalmente, hay una asociación de arqueólogos norteamericanos que trabaja en Africa que también tiene reuniones, pero sin revista. Las grandes sociedades (AAA, SAA y AAPA) publican los resúmenes de las comunicaciones presentadas en sus reuniones, pero no publican actas. Los prehistoriadores del Viejo
Mundo (y los paleoantropólogos) son una pequeña minoría, pero son muy activos, especialmente en sus grupos especializados y dentro de la SAA y la AAPA.

Naturalmente, los grandes temas son los del origen y evolución del hombre, la(s) migracion(es) fuera de África, la transición del Paleolítico Medio al Superior, las adaptaciones de los humanos en el Pleistoceno, el arte rupestre y mueble, la transición del Pleistoceno al Holoceno, el poblamiento de las Américas desde Siberia, el origen y expansión de la agricultura, la evolución y funcionamiento de las civilizaciones, etc. También en la actualidad hay mucha preocupación por los problemas metodológicos (tafonomía, naturaleza del registro, análisis faunístico, tecnología lítica y cerámica, etc.) y teóricos. El postprocesualismo todavía no ha afectado mucho al estudio de la Prehistoria del Viejo Mundo - especialmente la de los humanos del Paleolítico- en EE.UU. Sin embargo hay más interés ahora en la reconstrucción de aspectos sociales que en la "nueva arqueología" procesualista "clásica" de los años 1970. Esto es un buen signo y con ello el estudio de la Prehistoria en los EE.UU. se acerca más al del Viejo Mundo a la vez que éste adopta alguna de las características más interesantes del norteamericano.

\section{CONCLUSIONES}

A mi juicio, hay dos papeles importantes para los norteamericanos en la práctica de la investigación prehistórica en el Viejo Mundo:

1. Aportar ideas nuevas y distintas desde una perspectiva antropológica para complementar las perspectivas históricas y, a veces (como en el caso del estudio Bordesiano del Paleolítico en Francia) geológicas, que son más típicas (aunque no exclusivas) en el Viejo Mundo.

2. Montar proyectos de investigación interdisciplinares con objetivos muy bien definidos, a la vez con enfoques generalistas y con fines específicos, y con financiación adecuada.

El primer papel puede traer una dialéctica mutuamente fructífera con los colegas "nativos"; el segundo tiene que implicar una colabo-

T. P., 53, n. ${ }^{\circ} 1,1996$ 
ración auténtica, ya que ambas partes tienen mucho en lo que contribuir: los "nativos" incorporan una experiencia que difícilmente puede tener un norteamericano, pero éste puede aportar el característico vigor y, a veces, organización norteamericanos —además de sus ideas "geniales", "ingenuas" o simplemente "diferentes". Sin ofender a nadie, espero haber expuesto una descripción y una justificación adecuadas de la investigación prehistórica en el Viejo Mundo por parte de arqueólogos del Nuevo Mundo.

\section{AGRADECIMIENTOS}

Quisiera agradecer a mi alumno, Jon Orphal, que ha compilado las listas de las especialidades de los arqueólogos con intereses en el Viejo Mundo a partir de la guía de la SAA. También quisiera agradecer a mi colega, la directora de Trabajos de Prehistoria, la doctora María Isabel Martínez Navarrete, por haberme invitado a escribir esta nota (y por haber corregido mi castellano rudimentario) ¡Espero que no haya quedado completamente decepcionada!
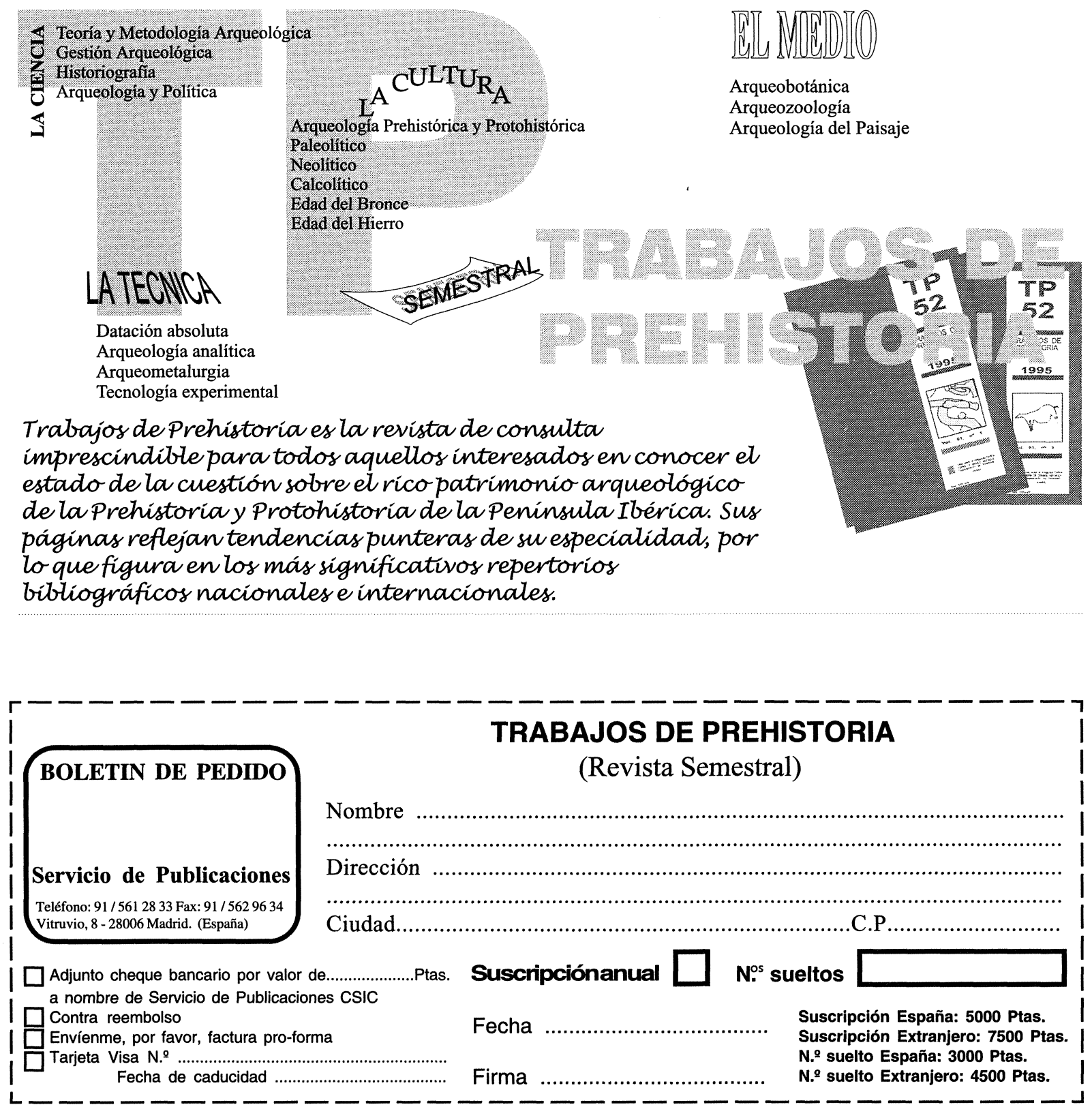

T. P., 53, n. ${ }^{\circ} 1,1996$ 